\title{
Water affinity to epitaxial graphene: the impact of
}

\section{layer thickness}

Cristina E. Giusca ${ }^{*}$, Vishal Panchal ${ }^{1}$, Martin Munz ${ }^{1}$, Virginia D. Wheeler ${ }^{2}$, Luke O. Nyakiti ${ }^{3}$, Rachael L. Myers-Ward ${ }^{2}$, D. Kurt Gaskill², Olga Kazakova ${ }^{1}$

${ }^{1}$ National Physical Laboratory, Hampton Road, Teddington, TW11 0LW, United Kingdom

${ }^{2}$ U.S. Naval Research Laboratory, Washington, DC 20375, United States of America

${ }^{3}$ Texas A\&M University, Galveston, TX 77553, United States of America

KEYWORDS: epitaxial graphene, surface potential, water adsorption, controlled environment, sensors

ABSTRACT: The sensitivity to water of one-, two- and three-layer epitaxial graphene (1, 2 and $3 \mathrm{LG}$ ) is examined in this study. We unambiguously show that graphene's response to water, as measured by changes in work function and carrier density, is dependent on its thickness, with $1 \mathrm{LG}$ being the most sensitive to water adsorption and environmental concentration changes. This is furthermore substantiated by surface adhesion measurements, which bring evidence that 1LG is less hydrophobic than 2LG. Yet, surprisingly, we find that other contaminants commonly present in ambient air have a greater impact on graphene response than water vapour alone. This study indicates that graphene sensor design and calibration to minimize or discriminate the effect of the ambient in which it is intended to operate are necessary to insure the desired sensitivity and reliability of sensors. The present work will aid in developing models for realistic graphene sensors and establishing protocols for molecular sensor design and development.

\footnotetext{
* To whom correspondence should be addressed. E-mail: cristina.giusca@npl.co.uk
} 
Although graphene has been shown to exhibit great potential for sensing applications $[1,2$, 3], no attention is paid to how the thickness of graphene affects the sensor response in terms of sensitivity, selectivity, response time or reproducibility. The different electronic structure of one-, two- and thicker graphene layers [4] suggests that the sensor response can be affected by domain thickness and we demonstrate this through subjecting graphene samples with welldefined number of layers to varying humidity levels. Since water is the most abundant dipolar adsorbate under ambient ${ }^{\dagger}$ conditions, significant effort has been dedicated to both theoretical $[5,6,7]$ and experimental $[8,9,10]$ investigations of water on graphitic surfaces in an attempt to elucidate the water-graphene interaction from an electronic structure perspective. While some density functional theory (DFT) calculations predicted the formation of a small energy gap of the order of 20-30 meV when graphene is fully covered with water molecules [16], other theoretical studies show that adsorbed water has very little effect on the electronic structure of graphene $[7,17]$. The unpredictable influence that environmental humidity exposure have on graphene devices processed and operated in ambient has also been highlighted by several previous experimental studies $[11,12,13,14,15]$. Nevertheless, there is currently a lack of studies concerned with quantitative aspects and reliable measures of changes in the electronic properties of graphene due to environmental humidity changes. Furthermore, studies of graphene exposed to various target gases (not commonly found in the ambient in the concentrations of interest) and ambient environment have been carried out on mechanically exfoliated graphene $[9,10,19]$, with very little work done on epitaxial graphene, which holds a practical route for many applications (e.g. optoelectronics, Quantum Hall effect metrology, high speed electronics), given its compatibility with wafer-scale processing techniques. Moreover, the intrinsic doping level information obtained from these transport measurements is generalized over the entire device and is not correlated with the exact morphology of graphene

\footnotetext{
${ }^{\dagger}$ Here, ambient refers to the typical work place or laboratory environment, that is, the air has not been specially processed or cleaned of impurities. 4.79For this study, ambient is the untreated air in the laboratory where the samples were investigated.
} 
in terms of domain thickness and the presence of local adsorbates that can influence the electronic properties of graphene.

In the current work, we employ scanning Kelvin probe microscopy (SKPM) to investigate the effect that water has on the electronic properties of epitaxial graphene, directly correlated with local structural information and electrical transport measurements, in order to provide a systematic evaluation of the impact of ambient exposure on epitaxial graphene, in accordance with its domain thickness. SKPM provides an electrical map of the surface that gives essential information on graphene thickness, layer-dependent distribution of charge, electrical potential and work function $[20,21,22]$. We study the influence of relative humidity variation $(\mathrm{RH}=10$ $70 \%)$ on the surface potential of one-, two- and tri-layer epitaxial graphene (1LG, 2LG and 3LG, respectively) on $\mathrm{SiC}$, as well as the effect that the change in environment, from ambient to vacuum and to high humidity levels, has on the electronic properties of the various graphene domains. Additionally, the water-graphene interaction is examined in terms of water adhesion to $1 \mathrm{LG}$ and $2 \mathrm{LG}$, with a view to correlating the surface potential with the degree of wettability of graphene domains of different thicknesses.

We unambiguously demonstrate that graphene domains of different thicknesses react differently to the change in environment and that the sensitivity to water, as measured by changes in work function and carrier density, increases with decreasing domain thickness, with 1LG being the most sensitive to water adsorption and change in environment. Understanding the role of 1,2 and $3 \mathrm{LG}$ and their response to various environments will impact the design and development of molecular sensors and will help to correctly model the response of a realistic sensor.

Epitaxial graphene films have been investigated by Raman spectroscopy, SKPM and adhesion measurements, with Hall bar devices for transport measurements fabricated using standard electron beam lithography. SKPM experiments on graphene films and transport measurements of graphene devices have been performed in ambient (for "ambient" definition 
see above), vacuum, nitrogen and increasing humidity levels, as described further below. Details of sample growth, device fabrication and transport measurements, SKPM, adhesion measurements and Raman spectroscopy measurements are given in Methods.

Raman spectroscopy: In order to evaluate the quality and number of constituent layers, Raman spectroscopy mapping has been carried out and provides evidence that the sample consists mainly of $1 \mathrm{LG}$ with continuous strips of $2 \mathrm{LG}$ formed along $\mathrm{SiC}$ terrace edges, as exemplified in Figure $1 \mathrm{a}$ and $1 \mathrm{~b}$, where G-band and 2D-band intensity maps of a $(15 \times 15) \mu \mathrm{m}^{2}$ area are presented.

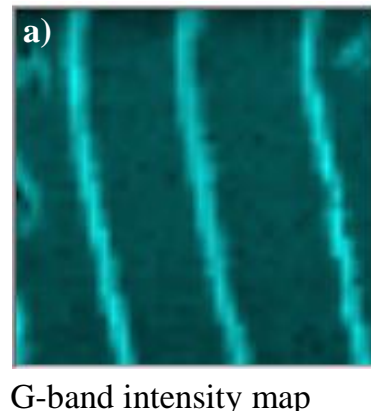

G-band intensity map

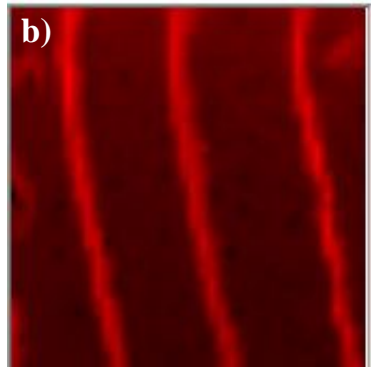

2D-band intensity map

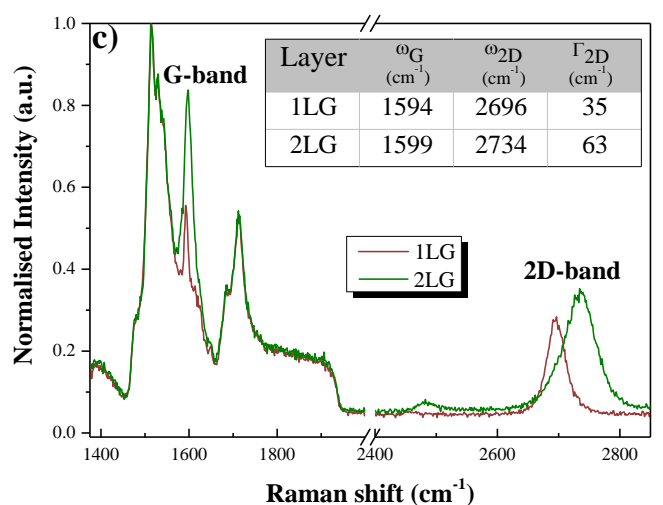

Figure 1: G-band (a) and 2D-band (b) Raman relative intensity maps of (15x15) $\mu \mathrm{m}^{2}$ area graphene obtained using 532-nm excitation wavelength. (c) Representative individual Raman spectra for 1LG and 2LG. In the G-band range the spectra are dominated by the SiC modes of the supporting substrate. Inset table shows the $G$ and $2 \mathrm{D}$ peak position, $\omega_{\mathrm{G}}$ and $\omega_{2 D}$, for $1 \mathrm{LG}$ and $2 \mathrm{LG}$, respectively, and the full-width-at-half-maximum $\Gamma$ for the 2D band. SiC contribution was not subtracted from the individual spectra to highlight that changes in peak positions and widths are only associated with graphene and not with SiC modes.

As illustrated in Figure 1c, representative individual Raman spectra for 1LG and 2LG display both the G- and 2D-modes typical to graphene, as well as the second order features of $\mathrm{SiC}$ in 
the range $1450-1750 \mathrm{~cm}^{-1}$. The G-band, associated with the doubly degenerate phonon mode vibrations at the center of the Brillouin zone, is located at $\sim 1594 \mathrm{~cm}^{-1}$ for $1 \mathrm{LG}$, but it becomes more intense, twice wider and shifts to higher wavenumbers for $2 \mathrm{LG}$. A similar trend is observed for the 2D-band of 2LG that displays a blue shift compared to that of 1LG, while becoming more asymmetric and almost doubling its width. Moreover, the shape of the 2D-band for $1 \mathrm{LG}$ can be perfectly fitted using a single Lorentzian peak, whereas four distinct components are required for that of $2 \mathrm{LG}$, as expected with the evolution of the electronic bands on the transition from one to two graphene layers [23].

Scanning Kelvin probe microscopy: SKPM experiments were conducted in an environmental chamber, where the atmosphere was controlled using the sequence: ambient (as defined above), vacuum, nitrogen and increasing humidity levels in nitrogen, on the same region of the sample and using the same scanning tip to ensure consistency of measurements. Topography was recorded simultaneously with surface potential images in all cases. As no modification of topography had been observed throughout the entire sequence of measurements, only the image recorded in ambient is shown for exemplification in Figure $2 \mathrm{a}$. A sequence of representative surface potential images of epitaxial graphene characterised by insitu SKPM under various environments (ambient 1, vacuum, nitrogen, humid conditions and ambient 2) is displayed in Figure 2 (b-1). The initial measurements were carried out in ambient (ambient 1), at room temperature $\left(\mathrm{T}=21^{\circ} \mathrm{C}\right)$ with no prior surface conditioning of the graphene sample. Following ambient exposure, the surface potential was measured in vacuum after the sample was annealed at $150^{\circ} \mathrm{C}$ to remove water and atmospheric adsorbates and subsequently cooled down to room temperature. The following step involved exposing the sample to nitrogen gas $(99.9995 \%$ purity) in order to bring the environmental chamber from vacuum to atmospheric pressure for surface potential measurements under humid conditions. The humidity exposure sequence was carried out in a stepwise manner, from $10 \% \mathrm{RH}$ to $70 \% \mathrm{RH}$, using $\mathrm{N}_{2}$ as a background gas. Finally, the sample was re-exposed to ambient (ambient 2) after 
the entire measurement cycle. Further details on exposure to various environments and measurement time are included in Methods.
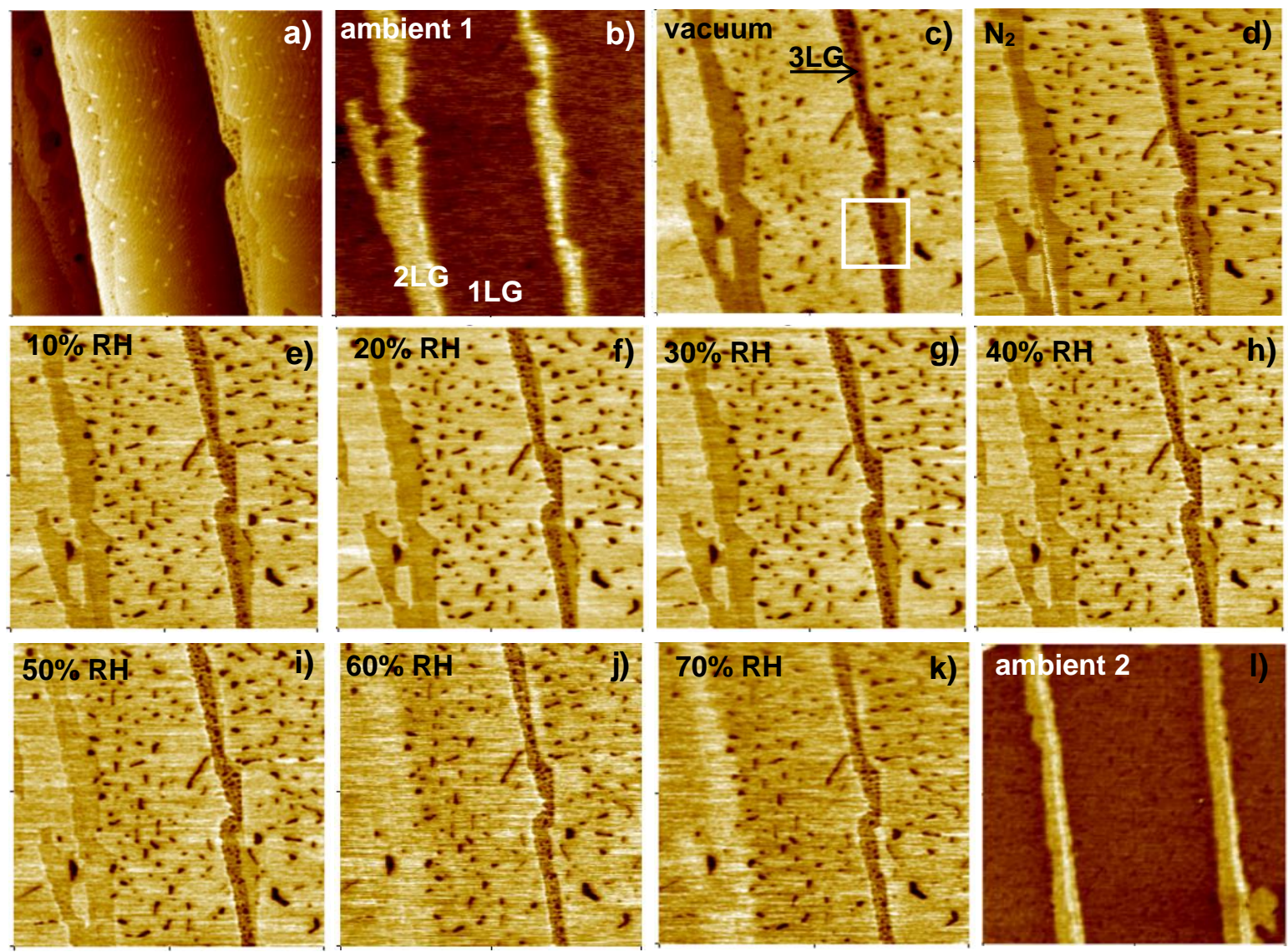

Figure 2: Topography (a) and sequence of surface potential images (b-k) collected on the same region of the graphene sample, while environmental conditions were changing from ambient to vacuum, nitrogen and to increasing levels of humidity in nitrogen. Image in (I), showing the surface potential after re-exposure to ambient, was taken on a different region of the sample due to a small offset induced by drift when venting the environmental chamber. The scan size is $(10 \times 10) \mu m^{2}$ for all images. Surface potential images were acquired simultaneously with topography images, but only one representative topography image is shown in (a). The surface potential maps are plotted on different $\mathrm{Z}$ scales to enhance difference in contrast. See Table S1 for the absolute values of surface potential for 1,2 and $3 L G$. 
Topography image displayed in Figure 2a reveals a terraced surface morphology with parallel edges consistent with typical SiC morphology, also showing the scarce presence of buffer layer regions as small, bright filamentary structures on terraces. While topography does not allow for a clear identification of the number of graphene layers, the surface potential map acquired simultaneously in ambient conditions (Figure 2b), shows regions with two main distinct contrast levels: a bright one, given by two parallel stripes, associated with $2 \mathrm{LG}$, superimposed on a dark contrast background of 1LG. This is furthermore highlighted by the corresponding histogram associated with the surface potential map (Figure 3a), displaying a bi-modal surface potential distribution, consistent with the number of layers revealed by Raman spectroscopy maps in Figure 1a. The histogram presents the surface potential distribution relative to 1LG, which was artificially assigned to $0 \mathrm{eV}$, in order to emphasise the surface potential difference between the layers. Peak deconvolution was carried out using Lorentzian shape components and layer thickness was designated in accordance with the domains contrast in the surface potential image, such that the most intense peak corresponds to the brightest contrast in the image.
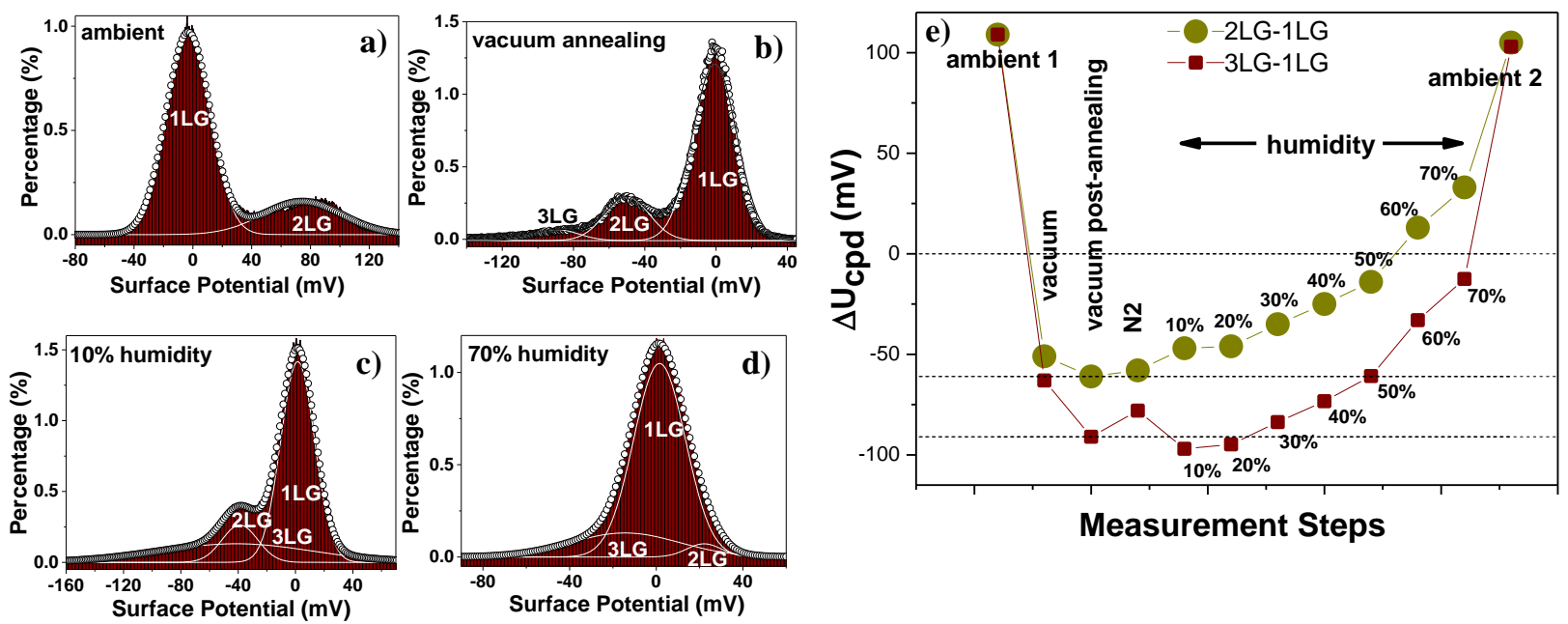

Figure 3: Histograms associated with surface potential maps shown in Figure 2, illustrating relative contact potential difference (UCPD) values between individual layers, 
are shown for ambient (a), vacuum annealing (b), low humidity (c), and high humidity (d) conditions, respectively. Histograms correspond to the area enclosed by the white square in Figure 2c. Curve fitting and layer thickness designation are described in text. (e) Sequence of contact potential difference $\left(\Delta \mathbf{U C P D}_{\mathrm{C}}\right)$ variations for $2 \mathrm{LG}$ and 3LG with respect to 1LG. Environmental conditions were changing in the following order: ambient, vacuum, nitrogen, increasing levels of humidity, from $10 \%$ to $70 \% \mathrm{RH}$, followed by reexposure to ambient. "Ambient 1" and "ambient 2 " represent the values recorded at the initial and the final step of the measurement cycle, respectively. The value of ambient humidity was $40 \%$ RH.

Based on the values derived for the contact potential difference in ambient and calibrated value for the tip work function, $\Phi_{t i p}=4.1 \mathrm{eV}$ (see Methods) [23], work function values for 1LG and 2LG have been calculated using $e \Delta U_{C P D}=\Phi_{t i p}-\Phi_{\text {surface }}$. The work function values obtained for ambient are $4.79 \mathrm{eV}$ for $1 \mathrm{LG}$ and $4.68 \mathrm{eV}$ for $2 \mathrm{LG}$. Previous SKPM studies in ambient report a work function value of $\sim 4.5 \mathrm{eV}$ for graphene $[24,25,26]$, that depends on the number of layers and can be tuned by ion irradiation-induced defects [25] or electric field effect within the range $4.5-4.8 \mathrm{eV}$ for $1 \mathrm{LG}$ and $4.65-4.75 \mathrm{eV}$ for $2 \mathrm{LG}$ [26]. Following ambient exposure, the chamber was pumped for $\sim 16$ hours to $\mathrm{P}=1 \times 10^{-5}$ mbar and subsequently annealed at $150^{\circ} \mathrm{C}$ for 2 hours, under vacuum conditions, in order to remove impurities and air adsorbates, including water. This procedure always results in a reproducible surface, as suggested by the relatively constant surface potential values obtained for multiple measurement cycles [18], and is consistent with previous experiments reporting reduction of p-doping following vacuum annealing of graphene $[12,27]$. Contrast inversion of the surface potential (with respect to surface potential observations in ambient conditions) is observed upon vacuum annealing (Figure 2c) and the related histogram (Figure 3b), where 1LG now displays brighter contrast than 2LG. Interestingly, 3LG becomes visible in the surface potential map under 
vacuum and shows an even darker contrast relative to 1 LG than $2 \mathrm{LG}$. Additionally, the buffer layer features (hardly distinguishable on the surface potential image under ambient conditions) have also become visible in vacuum. The work function value of $1 \mathrm{LG}$ obtained in vacuum, $(4.31 \pm 0.02) \mathrm{eV}$ agrees well with the value of $4.34 \mathrm{eV}$ measured by A. Tadich et al. on clean epitaxial graphene [28].

In the next set of experiments, after vacuum annealing the sample was first exposed to dry nitrogen at atmospheric pressure and then to varying humidity levels, ranging between $10 \%$ and $70 \% \mathrm{RH}$. Change in contrast is observed in the surface potential images for $2 \mathrm{LG}$ and $3 \mathrm{LG}$ relative to $1 \mathrm{LG}$ with the change of environment and $\mathrm{RH}$, as seen in Figure 2 e-k, whereas associated topography images (not shown) remain unaffected.

The summary of these experiments is presented in sequential order in Figure $3 \mathrm{e}$, illustrating the contact potential difference $\triangle U_{C P D}{ }^{2-1}$ and $\triangle U_{C P D}{ }^{3-1}$ measured for $2 \mathrm{LG}$ and $3 \mathrm{LG}$ relative to 1LG, respectively. $\triangle U_{C P D}{ }^{2-1}$ and $\triangle U_{C P D}^{3-1}$ values for each environment (ambient, vacuum, nitrogen, $\mathrm{RH}$ in $\mathrm{N}_{2}$ ) have been extracted from associated histograms of surface potential distribution, as shown in Figure 3a-d. The surface potential values for 1, 2 and 3LG for each environment are presented in Table S1 in the Supporting Information section. The plot in Figure 3e shows that the contact potential difference measured by SKPM between the 2LG and 1LG, $\triangle U_{C P D}^{2-1}$ changes sign, from $\sim 109 \mathrm{mV}$ in ambient to $\sim-51 \mathrm{mV}$ in vacuum, which decreases further to $\sim-61 \mathrm{mV}$ upon annealing in vacuum. This behaviour is consistent with gradual desorption of loosely bound species, such as water and other electron withdrawing adsorbed species (p-dopants), which appear to derive from the ambient, during pumping. However, pumping alone is not sufficient to remove all environmental dopants (or at least for the 16 hours used in this study). As shown by the further decrease in surface potential, heat treatment of the sample in vacuum (for 2 hours, at $150^{\circ} \mathrm{C}$ ) seems to be more effective in removing environmental adsorbates than pumping alone. Our observations are in agreement with previous reports showing that the characteristics of four-terminal graphene devices 
improve upon pumping and heat treatment, resulting in a smaller hysteresis in the electric field effect behaviour, charge neutrality point closer to zero back-gate voltage and larger resistance at the charge neutrality point [12], [40].

Introducing $\mathrm{N}_{2}$ into the chamber after vacuum annealing the sample and prior to humidity exposure, shows a slight increase of $\Delta U_{C P D}{ }^{2-1}$ to $\sim-58 \mathrm{mV} . \mathrm{N}_{2}$ is an inert gas at room temperature, and it should not produce any change in surface potential, however contaminants can be present on the inner walls of the non-metallic tube carrying $\mathrm{N}_{2}$ into the chamber, even though flushing the line with $\mathrm{N}_{2}$ gas had been carried out several times. Following this step, the absolute value of $\triangle U_{C P D}{ }^{2-1}$ gradually increases with increasing $\mathrm{RH}$, passes through 0 at $\sim 55 \%$ $\mathrm{RH}$ and reaches positive values for $60 \%$ and $70 \% \mathrm{RH}$ levels. The work function values obtained for $70 \% \mathrm{RH}$ are $4.59 \mathrm{eV}$ for $1 \mathrm{LG}$ and $4.55 \mathrm{eV}$ for $2 \mathrm{LG}$. As illustrated in Figures $2 \mathrm{j}$ and $2 \mathrm{k}, 2 \mathrm{LG}$ shows inverted contrast at $60 \%$ and $70 \% \mathrm{RH}$, although not reaching the level observed initially under ambient conditions.

A similar trend is observed for $\triangle U_{C P D}^{3-1}$ measured for $3 \mathrm{LG}$ with respect to $1 \mathrm{LG}$, as illustrated in Figure 3e, although with more depressed values that do not cross the zero-level to invert the contrast for $3 \mathrm{LG}$.

It is important to point out that the surface potential (and consequently the work function) values reproducibly return to the initial level when the sample is re-exposed to ambient following the measurement cycle in the various environments. The work function variation with the change in environment for 1, 2 and $3 \mathrm{LG}$ is displayed in Figure 4 and the respective work functions are summarised in Table S1. The work functions have been obtained based on the absolute values of surface potential for each individual layer, and were also used to plot the surface potential differences of 2LG and 3LG relative to 1LG shown in Figure 3. 


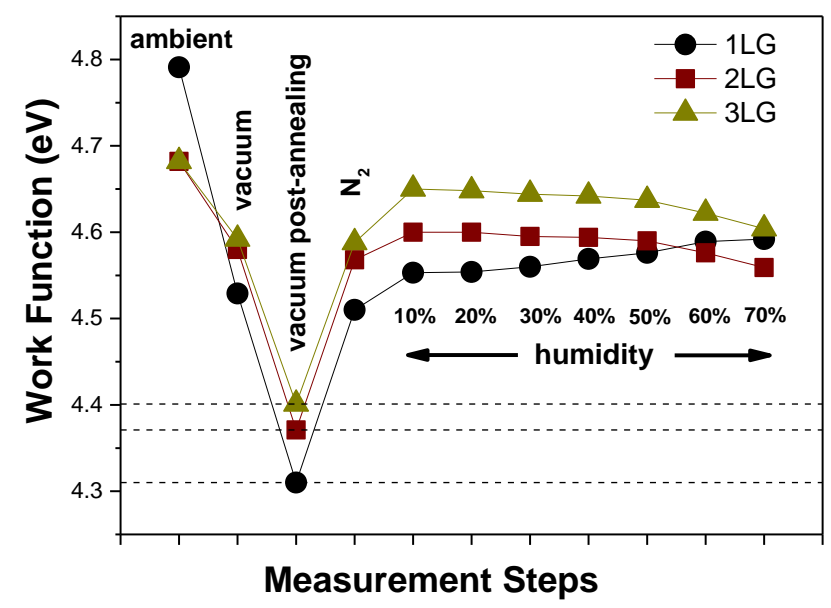

Figure 4: Work function variation for 1, 2 and 3LG with the change in environment.

The work function calculations for 1, 2 and $3 \mathrm{LG}$ assumed a constant work function for the tip, as no changes in free amplitude and fundamental oscillating frequency of the tip were detected with the change in humidity. Previous SKPM studies on graphite also found that the surface potential contribution of water adsorbed on the tip is significantly smaller than that adsorbed on the sample surface [29].

It can be seen that the work function values for 1, 2 and $3 \mathrm{LG}$ decrease with respect to ambient when the sample is annealed under vacuum, as expected with removal of p-dopants. A greater change in work function is observed for $1 \mathrm{LG}$ than for $2 \mathrm{LG}$ and $3 \mathrm{LG}$ on the transition from ambient to vacuum, indicating that $1 \mathrm{LG}$ is more sensitive to the change of environments than 2 and 3LG. When exposing the sample to increasing humidity levels, the work function of 1LG increases with humidity (10 - 70\% RH). Interestingly, the work functions of 2LG and 3LG reveal a reverse trend compared to $1 \mathrm{LG}$, showing a slow decrease in work function as the humidity increases. The work function values coincide for $1 \mathrm{LG}$ and $2 \mathrm{LG}$ at $\sim 50 \% \mathrm{RH}$ and for $1 \mathrm{LG}$ and $3 \mathrm{LG}$ at about $\sim 70 \% \mathrm{RH}$ (Fig. 4), indicating that the Fermi level is changing at a different rate for 1, 2 and 3LG with water adsorption. In summary, the SKPM results show that different layer numbers result in quantitative differences in water adsorption and change in 
environment. Furthermore, these differences are linked to changes in the physical and chemical properties of graphene's surface.

Electrical transport measurements: Surface potential measurements have been corroborated with electrical transport data recorded on graphene Hall bar devices containing 1LG and 2LG crosses of 760-nm width. The morphology and thickness of the device was determined using SKPM (details concerning device fabrication and transport measurement are presented in Methods). Transport measurements have been performed following a similar sequence, from ambient to vacuum, nitrogen and increasing $\mathrm{RH}$ in $\mathrm{N}_{2}$, as for the SKPM experiments summarised in Figure 2. As shown in Figure 5, the carrier density (n-type) for both $1 \mathrm{LG}$ and $2 \mathrm{LG}$ increases in vacuum with respect to the values observed in ambient.
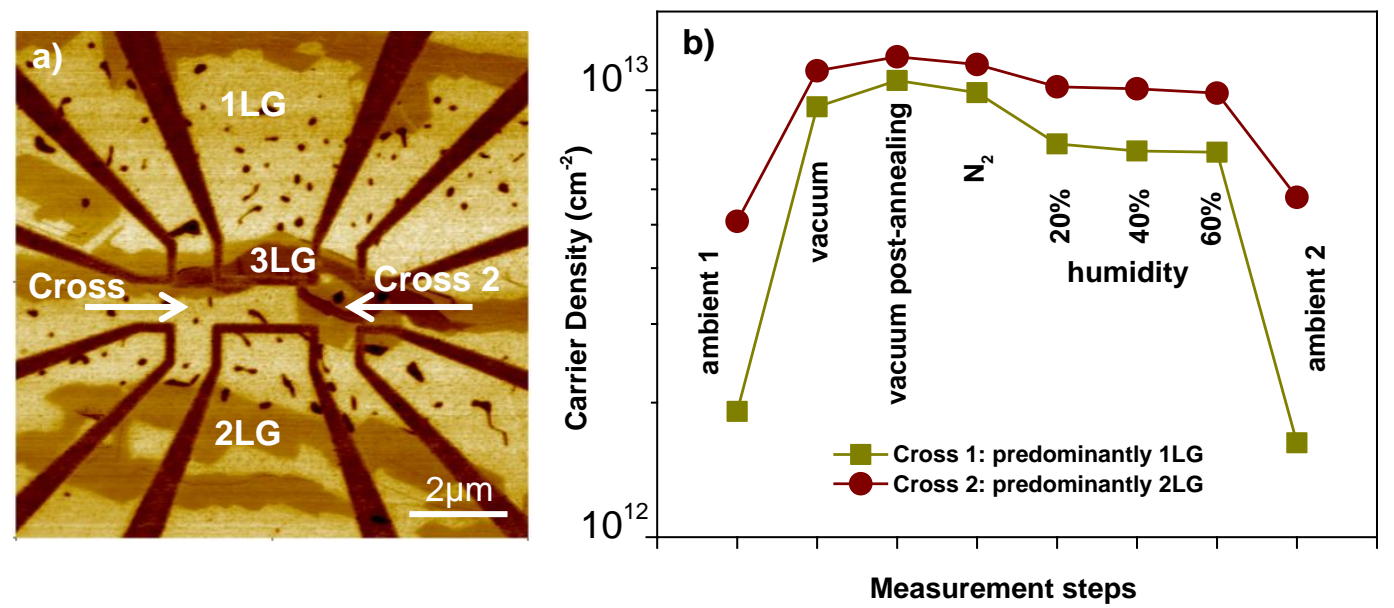

Figure 5: (a) Surface potential image of the device used for transport measurements taken in vacuum, after temperature annealing at $150{ }^{\circ} \mathrm{C}$. (b) Carrier density (n-type) extracted from transport measurements performed with the sample going through a similar sequence as for SKPM experiments in Figure 2.

The change in carrier density is more pronounced for $1 \mathrm{LG}$ compared to $2 \mathrm{LG}$, a trend that is followed throughout the entire sequence of measurements, although the largest carrier change occurs with the ambient-vacuum transition as also observed for the work function trend. A 
higher carrier density is determined for $2 \mathrm{LG}$ compared to $1 \mathrm{LG}$ for the entire measurement cycle. The environmental change in the carrier density follows the same trend as for the absolute values of surface potential for 1 and 2LG (shown in Supplementary Information section) and is generally in agreement with the recent work by Yang et al. treating doping by molecular adsorbates in ambient for epitaxial graphene [40]. Figure 5b illustrates that values of the carrier density at $\mathrm{RH}=20-60 \%$ are significantly different from the values during ambient exposure for both 1 and 2LG.

Although the transport measurements were performed at a relatively high DC bias current ( $\mathrm{I}_{\text {bias }}$ $=50 \mu \mathrm{A})$, we observed no real change in the transport properties of the device when studied in the range 10-100 $\mu \mathrm{A}[41]$. While there is no measurable increase of the temperature detected at $\mathrm{I}_{\mathrm{bias}}=50 \mu \mathrm{A}$, a small temperature increase of $0.2^{\circ}$ was measured in the vicinity of the device at $\mathrm{I}_{\mathrm{bias}}=100 \mu \mathrm{A}$. Both these results together with a good thermal conductance of SiC confirm that there are no real inductive heating effects for micron-wide epitaxial graphene devices on $6 H$ $\operatorname{SiC}(0001)$.

Consistent with the SKPM results, the transport measurements show that the water adsorption behaviour is dependent on the layer number and that $1 \mathrm{LG}$ is more affected than $2 \mathrm{LG}$ upon exposure to $\mathrm{RH}$, pointing to a different impact that surface absorbed water has on $\mathrm{E}_{\mathrm{F}}$ for $1 \mathrm{LG}$ relative to $2 \mathrm{LG}$.

The transport data and the work function values determined by SKPM are used to schematically illustrate the energy band diagrams of $1 \mathrm{LG}$ and $2 \mathrm{LG}$ in Figure 6, where only the transitions from ambient to vacuum and to highest humidity level $(\mathrm{RH}=70 \%$ in the transport measurements) are presented for simplicity. 
a) Ambient

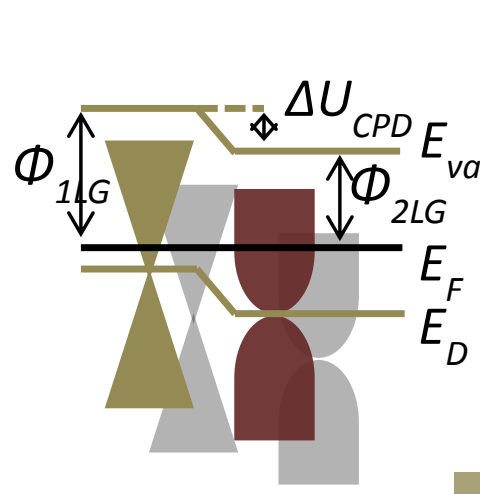

b) Vacuum

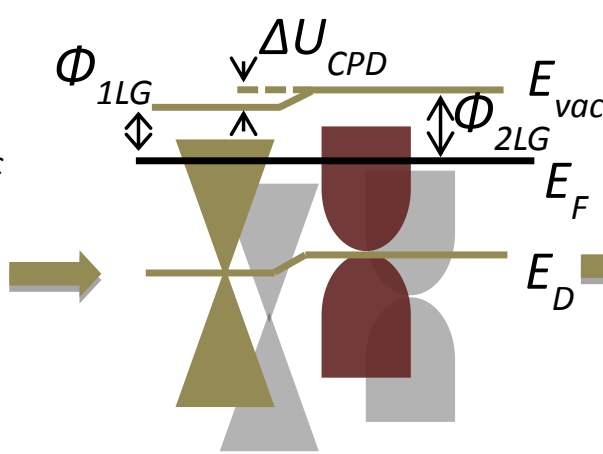

c) $70 \% \mathrm{RH}$

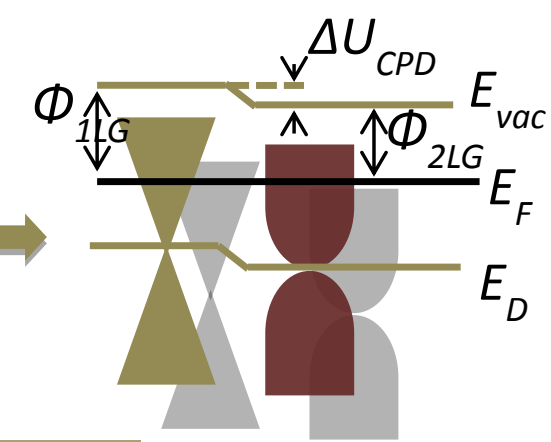

All environments: $n_{e}(1 \mathrm{LG})<n_{e}(2 \mathrm{LG})$

1LG \& 2LG: $\boldsymbol{n}_{e^{\text {ambient }}}<\boldsymbol{n}_{e^{70 \%} \mathrm{RH}}<\boldsymbol{n}_{e^{\mathrm{vacuum}}}$

Figure 6: Schematic energy band diagram for 1LG and 2LG, illustrating respective changes in work function upon exposure to (a) air, (b) vacuum and (c) high humidity $(\mathbf{R H}=\mathbf{7 0} \%)$.

A higher carrier (electron) density for both $1 \mathrm{LG}$ and $2 \mathrm{LG}$ is observed in vacuum compared to their respective ambient values, consistent with the work function values determined by SKPM. As discussed previously, the observed increase of the electron concentration in both 1LG and 2LG on ambient to vacuum transition is due to the desorption of environmental p-dopants and the increase for $1 \mathrm{LG}$ is larger than that of $2 \mathrm{LG}$.With the addition of water, the sample shows an increase in the work function for both $1 \mathrm{LG}$ and $2 \mathrm{LG}$ relative to vacuum, consistent with an increased p-doping concentration and lower resulting carrier density for both 1LG and 2LG compared to the values measured in vacuum (Figure 6c). The difference in work function for 1LG on vacuum-humidity transition is larger than for $2 \mathrm{LG}$. However, the doping concentration obtained for high $\mathrm{RH}$ levels does not reach the concentration observed in ambient, indicating that other factors in the ambient, in addition to water, are p-dopants and affect the surface potential of graphene. This observation together with qualitative differences in carrier densities for 1 and 2LG in all studied environments (Figure 5b) indicate higher sensitivity of 1LG to 
water vapour absorption as compared to $2 \mathrm{LG}$. It is important to point out that $U_{C P D}$ values of 1LG and 2LG reproducibly restored to the initial values whenever re-exposing the sample to ambient, i.e. after vacuum or humidity treatment, as indicated in Figure $3 \mathrm{e}$ and also supported by the transport data in Figure 5b. These observations demonstrate that the properties of graphene are strongly influenced by external doping imposed by atmospheric adsorbates. The same trend exhibited by $2 \mathrm{LG}$ with the change in environment and exposure to humidity is also observed for 3LG.

In an attempt to clarify whether oxygen from ambient air has a greater effect on graphene than humidity, the sample was subjected to dry synthetic air containing $20 \%$ oxygen and $80 \%$ nitrogen, following pumping and annealing at $150^{\circ} \mathrm{C}$ under vacuum. No apparent effect of oxygen on the surface potential of $1 \mathrm{LG}, 2 \mathrm{LG}$ or $3 \mathrm{LG}$ was noted and the contact potential difference $U_{C P D}$ decreased only slightly in absolute value by $\sim 3 \mathrm{mV}$ compared to its vacuum value. The combined effect of oxygen and water was noted by previous works to be responsible for the hole doping of graphene [24, 27, 39]. We further tested the effect of humidity on the surface potential and transport data using synthetic air as a background gas (not shown). However, we observed the same trend as for humidity using $\mathrm{N}_{2}$ gas (Fig. 3, 4 and 5), still not accounting for the full difference we observe relative to the starting ambient point.

Adhesion mapping: Since our study indicates a greater sensitivity of water for 1LG than 2LG, we further examine how the surface potential data correlates with the wetting behaviour of graphene domains of different thicknesses. For this purpose, adhesion mapping has been carried out by recording force-distance curves at every pixel on a preselected region on the sample. The experiments have been performed with the sample immersed in de-ionised (DI) water (resistivity $18.2 \mathrm{M} \Omega \cdot \mathrm{cm}$ ) in order to avoid the effect of the water contamination layer usually present on surfaces scanned in ambient, giving rise to a liquid capillary between the probe and the sample [30] that is known to cause relatively large attractive interactions. In the present work, OTS functionalized tips have been used to map the spatial variation of attractive 
forces between hydrophobic tips and graphene in DI water. The resulting adhesion map, illustrated in Figure 7, clearly evidences the presence of 1LG and 2LG domains, consistent with the surface potential measurements presented in Figure 2.
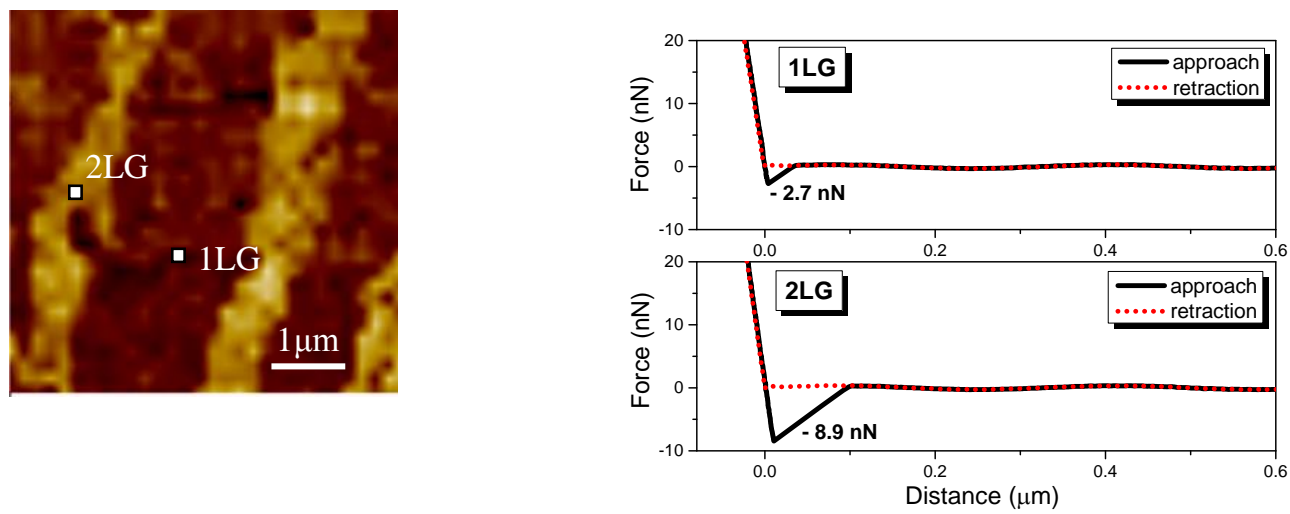

Figure 7: a) Adhesion map obtained on the sample immersed in DI water, using OTS functionalised probe. b) Associated force-distance curves extracted from selected locations on 1LG and 2LG domains.

Test samples with a pattern of hydrophobic OTS areas and hydrophilic gold areas confirmed the larger adhesion force on hydrophobic areas (see Supplementary Information) when using OTS functionalized tips. This indicates that a larger adhesion force is measured on more hydrophobic areas of the sample (i.e. $2 \mathrm{LG}$ ), due to the hydrophobic interaction, compared to less hydrophobic areas (i.e. 1LG). As highlighted in the force-distance curves in Figure $7 \mathrm{~b}$, a larger adhesion force is consistently measured on 2LG as compared to 1LG domains, suggesting that $1 \mathrm{LG}$ is less hydrophobic. Attractive forces of the order of $(7.6 \pm 1.6) \mathrm{nN}$ have been measured for $2 \mathrm{LG}$ domains and $(4.1 \pm 1.4) \mathrm{nN}$ for $1 \mathrm{LG}$. In humid air, the lower hydrophobicity is associated with a greater capillary force on $1 \mathrm{LG}$ compared to $2 \mathrm{LG}$ and is consistent with current SKPM and electrical transport observations of enhanced water sensitivity for $1 \mathrm{LG}$ relative to $2 \mathrm{LG}$. 
Discussion: Overall, both SKPM and transport measurements show that the thickness of graphene results in quantitative differences in water adsorption, that are linked to changes in graphene's surface physical and chemical properties. These properties change more for 1LG than for 2 and $3 \mathrm{LG}$ and water adsorption is greater on 1LG than on thicker graphene.

The lack of sensitivity of 2 and $3 \mathrm{LG}$ relative to $1 \mathrm{LG}$ is most likely due to the different electronic structure of single and multilayer graphene as shown by previous studies demonstrating that the electronic band dispersion near the Fermi level, and consequently the nature of the charge carriers, is highly sensitive to the number of layers, the stacking geometry and the interlayer interaction $[35,36,37]$. The $\pi$ bands of a single graphene layer do not exhibit dispersion with the out-of-plane electron momentum. However, each added layer affects the topology of the $\pi$ bands, and an additional band occurs due to the interaction between layers, as shown by angle-resolved photoemission spectroscopy (ARPES) [36]. From ARPES measurements of epitaxial graphene on $\mathrm{SiC}$, Ohta et al. extracted tight binding parameters, including interlayer hopping integrals and $\mathrm{E}_{\mathrm{F}}=440,300,210$, and $150 \mathrm{meV}$ for $1,2,3$ and 4 LG, respectively [36]. Since the total sheet charge density did not change much, it was concluded that interlayer screening results in a charge redistribution, with an effective screening length of 1.4 and $1.9 \AA$ for 3 and 4 LG, respectively, that increases further for thicker layers and graphite. Furthermore, DFT calculations showed that water molecules are less bound to $2 \mathrm{LG}$ than to $1 \mathrm{LG}[16]$, indicating a binding energy of $1.30 \mathrm{eV}$ per water molecule for $2 \mathrm{LG}$, compared to $1.94 \mathrm{eV}$ for $1 \mathrm{LG}$.

Both SKPM and transport measurements show that neither water nor oxygen alone has a dominating effect on the electronic properties of graphene. The same can be inferred about their combined contribution. But, since the case of the ambient exposure is so different from the controlled exposures using high purity gases, the implication is that some other (unknown) impurity (on their own or in combination with water and oxygen) is responsible for the difference that is measured in ambient relative to high humidity environment. Several previous 
theoretical studies showed that adsorbed water has very little effect on the electronic structure of graphene and that physisorption is generally observed at the water-graphene interface $[7,17$, 19]. It was shown by DFT calculations [17] that for small water clusters, charge transfer between graphene and water is very small and does not influence the density of charge carriers due to the fact that water molecules orient their dipole moments in opposite directions, so that they cancel on average. The discrepancy with experimental studies showing doping effects due to water adsorbates is likely to originate from the effect of the supporting substrate, as most of these studies use exfoliated graphene on $\mathrm{SiO}_{2}$ substrates. The substrate surface quality and chemical cleanliness were shown to play a crucial role for observation of doping by water adsorbates on graphene [19]. This indicates that more complex mechanisms might be involved than the simple interaction of graphene with single water molecules, for example, through the water-substrate interaction, as shown by Wehling et al. [19]. The dipole moments of water adsorbates can shift the substrate impurity bands and change their hybridisation with the graphene bands, leading to doping of graphene on thermally oxidised silicon substrates [19]. It was also postulated that oxygen and water adsorbates compensate for negatively-charged impurities in the supporting $\mathrm{SiO}_{2} / \mathrm{Si}$ substrate: for graphene sheets consisting mainly of p-type regions (corresponding to negatively-charged impurities in the substrate), the adsorbate layer tends to screen the substrate impurities and assists in mitigation of carrier scattering [9]. However, it is not clear whether the $\mathrm{SiC}$ substrate or the buffer layer at the graphene/SiC interface, controlling the electrostatic conditions of epitaxial graphene, play a similar role in mediating the interaction of water with epitaxial graphene.

Apart from water and oxygen responsible for the hole doping of graphene, the difference in the carrier density and work function values of graphene in ambient relative to high humidity suggests that other p-type doping contaminants commonly present in air have an additional effect on graphene. Likely candidates could be volatile hydrocarbon compounds that have been shown to significantly affect the wettability of graphitic surfaces through a large change in the 
water contact-angle with graphene, i.e. from $37^{\circ}$ for a clean surface to about $80^{\circ}$ for a hydrocarbon-contaminated one [31].

Conclusions: In summary, the electronic properties of graphene in controlled humidity environment have been examined in this study. The study shows that graphene's physical and chemical properties are sensitive to water vapour. Moreover, graphene's response to water and change in environment is strongly thickness dependent, with different layer numbers resulting in quantitative differences in water adsorption. The differences in water adsorption are linked to changes in graphene's physical and chemical properties. The results indicate increased water sensitivity with decreasing graphene thickness, with 1LG being the most affected by water and the change in environment. This observation is consistent with adhesion mapping measurements indicating that $1 \mathrm{LG}$ is less hydrophobic than $2 \mathrm{LG}$. The study shows that work function and carrier density of 1,2 and $3 \mathrm{LG}$ restore to the initial ambient values after the sequence of exposures involving vacuum and RH. The change in work function and carrier density with the change in environment is more pronounced for $1 \mathrm{LG}$ than for $2 \mathrm{LG}$, with $2 \mathrm{LG}$ consistently showing higher carrier density than 1LG for all examined environments. The RH exposure demonstrates a doping effect (p-type) that partly compensates the intrinsic n-type nature of the sample, as the overall carrier density decreases with increasing RH exposure. The work function of $1 \mathrm{LG}$ increases with increasing humidity level and the change in work function and doping is different in $1 \mathrm{LG}$ and $2 \mathrm{LG}$ for the same RH exposure.

Unexpectantly, our study finds that other contaminants commonly present in ambient air (such as volatile organic compounds or trace impurity gases) have a significant effect on the work function and doping of graphene. This is because the observed changes in work function and doping far exceed the changes found using pure Nitrogen and Oxygen gases, or via introduced humidity created from pure water vapour. This observation implies that real sensors need to be made immune to the effects of humidity and other constituents in the ambient, as 
selectivity is an essential ingredient of graphene sensors designed to work in in the ambient. Hence, additional experiments to understand the response to environmental effects are required so that realistic models can be formulated to insure the desired sensitivity of the sensor to the target molecule is obtained. Our work will provide a foundation for developing models for realistic sensors which could be extended to sensor designs that deliberately minimize the effects of the ambient.

\section{Methods}

1. The substrate (II-VI, Inc.) was ca. $8 \times 8 \mathrm{~mm}^{2}$ of semiinsulating (0001)6H-SiC (resistivity $\left.>10^{10} \mathrm{ohm} \mathrm{cm}\right)$ misoriented $\sim 0.05^{\circ}$ from the basal plane mainly in the (11-20) direction. Graphene was synthesized via $\mathrm{Si}$ sublimation from $\mathrm{SiC}$ using an overpressure of an inert gas. The substrates were etched in $\mathrm{H}_{2}$ at 200 mbar using a ramp from room temperature to $1580^{\circ} \mathrm{C}$ to remove polishing damage. At the end of the ramp, the $\mathrm{H}_{2}$ was evacuated and Ar added to a pressure of 100 mbar (the transition takes about 2 minutes). The graphene was then synthesized at $1580^{\circ} \mathrm{C}$ for $25 \mathrm{~min}$ in the Ar. Afterwards, the sample was cooled in $\mathrm{Ar}$ to $800^{\circ} \mathrm{C}$ [38]. Two or more layers of graphene formed in this fashion are known to be Bernal stacked [36].

2. Raman spectroscopy: Raman intensity maps were obtained using a Horiba Jobin-Yvon HR800 System. A 532-nm excitation was focused onto the sample through a 100x objective with $1 \mathrm{~mW}$ power incident and data were taken with a spectral resolution of $(3.1 \pm 0.4) \mathrm{cm}^{-1}$ and $\mathrm{XY}$ resolution of $(0.4 \pm 0.1) \mu \mathrm{m}$. The raw data were normalised with respect to the maximum of the two-phonon mode of $\mathrm{SiC}$ at $\sim 1514 \mathrm{~cm}^{-1}$.

3. Scanning Kelvin probe microscopy (SKPM) experiments in ambient, vacuum, $\mathrm{N}_{2}$ and humidity exposure were conducted on an NT-MDT NTEGRA Aura SPM system, using Bruker highly doped Si probes (PFQNE-AL) with a force constant $\sim 0.9 \mathrm{~N} / \mathrm{m}$ and resonant frequency of $\mathrm{f}_{0} \sim 300 \mathrm{kHz}$. Frequency-modulated SKPM (FM-SKPM) technique operated in a single pass mode has been used in all measurements. FM-SKPM operates by detecting the force gradient 
$(\mathrm{dF} / \mathrm{dz})$, which results in changes to the resonance frequency of the cantilever. In this technique, an $\mathrm{AC}$ voltage with a lower frequency $\left(\mathrm{f}_{\bmod }=3 \mathrm{kHz}\right)$ than that of the resonant frequency of the cantilever is applied to the probe, inducing a frequency shift. The feedback loop of FM-KPFM monitors the side modes, $f_{0} \pm f_{\text {mod }}$, and compensates the mode frequency by applying an offset DC voltage which is recorded to obtain a surface potential map. Since FMKPFM detects the force gradient using the frequency shift, it can achieve spatial resolution of $<20 \mathrm{~nm}$, which is limited only by the tip diameter, enabling the determination of the number of graphene layers of the sample with great accuracy.

For 1, 2 and 3LG work function calculations, the work function of the SKPM tip was determined using calibration against a gold reference sample. The work function of the gold sample was measured using ultra-violet photoelectron spectroscopy [32]. The value obtained for the work function of the tip is $(4.100 \pm 0.003 \mathrm{eV})$. It is important to note that a different value for the work function of the tip, although changing the absolute value of work functions we report for 1, 2 and $3 \mathrm{LG}$, would nevertheless result in the same trend that we observe upon the change in environment. The errors quoted in the manuscript for the work functions of 1,2 and 3LG have been determined from the standard deviation of the surface potential line profiles.

SKPM under different environments: FM-SKPM measurements in ambient air and vacuum ( $\mathrm{P} \sim 1 \times 10^{-5}$ mbar) were performed as described above. Measurements under vacuum were performed after the sample cooled down to room temperature following the sample annealing at $150^{\circ} \mathrm{C}$. Controlling the SPM chamber environment to $\mathrm{N}_{2}$ and synthetic air simply required regulating the gas flow using an inlet valve. For humidity exposure, RH was increased in a step-wise manner from $10 \%$ to $70 \%$ and the surface potential measurements were carried out immediately after the chamber reached the target humidity level and the time required for equilibrium to be established at low RH: $\sim 5 \mathrm{mins}$, and up to $\sim 30 \mathrm{mins}$ for $70 \% \mathrm{RH}$. The measurement time to acquire one complete scan is $15 \mathrm{~min}$. For each RH step, subsequent 
measurements were taken up to $1 \mathrm{~h}$ following each exposure and showed no change in surface potential, suggesting that the humidity effect was relatively quickly saturated on the surface of the graphene (less than 5 min. for $10 \%$ RH exposure).

4. Adhesion mapping: Employing a Cypher AFM system (Asylum Research, CA), the adhesion forces between chemically functionalized AFM probes and graphene surfaces were measured. The AFM system was fitted with a superluminescent diode to minimize signal oscillations resulting from optical interference of a non-zero fraction of light reflected off the sample surface with the light reflected off the cantilever. The AFM cantilevers used were of type CSC38 with aluminium reflective coating (Mikromasch Europe, Germany). Of the three rectangular silicon cantilevers of different lengths and spring constants available on a CSC38 chip, either cantilevers of $\sim 300$ or $\sim 350 \mu \mathrm{m}$ length were used. Typical spring constant values specified by the manufacturer are $\sim 50$ and $\sim 30 \mathrm{pN} / \mathrm{nm}$, respectively. Measured values of the spring constant were in the range of $\sim 80$ to $200 \mathrm{pN} / \mathrm{nm}$ and $\sim 80$ to $150 \mathrm{pN} / \mathrm{nm}$, respectively. To allow hydrophobic behavior of the AFM tip, the cantilevers were functionalized with octadecyltrichlorosilane (OTS). Prior to immersing into a solution of OTS in toluene, the cantilevers were treated in a UV/ozone cleaner for 20 min. Immediately after the immersion, the cantilevers were washed three times, in chloroform, acetone and again in chloroform. The concentration of the OTS-toluene solution was $\sim 10^{-3} \mathrm{~mol} / \mathrm{L}$. The AFM force measurements were undertaken in de-ionized (DI) water, by immersing the cantilever into a drop of $\sim 80 \mu \mathrm{L}$ volume on top of the graphene sample. The force-displacement curves were run at a scan rate of $\sim 0.81 \mathrm{~Hz}$ and a $\mathrm{z}$-velocity of $\sim 1.93 \mu \mathrm{m} / \mathrm{s}$. They were recorded over arrays of points and the magnitude of the pull-off peaks occurring upon retraction of the probe was analyzed. Typically, the pixel number of the resulting force maps was $64 \times 64$. To check the functionalization of the cantilevers and to characterize related adhesion contrasts, test samples with an array of gold squares on top of a silicon wafer surface were prepared. 
5. Device fabrication: Graphene devices in Hall bar geometry were fabricated from epitaxial graphene on Si-face $6 H$-SiC with electron beam lithography using Poly(methyl methacrylate) and ZEP520 (ZEON Chemicals) positive resists (thickness: 250 and $200 \mathrm{~nm}$, respectively), oxygen plasma etching (one minute) and electron beam physical vapour deposition of $\mathrm{Cr} / \mathrm{Au}(5 / 100 \mathrm{~nm})$. Graphene samples used for device fabrication were from the same batch of the graphene films used for the SKPM described in the paper. Further details on the fabrication process are reported in Ref. [33]. Using these fabrication processes, a $760 \mathrm{~nm}$ wide Hall bar device with cross 1 covered by 1LG ( 70\%) and 2LG ( 30\%), cross 2 covered by $2 \mathrm{LG}(\sim 70 \%)$ and $3 \mathrm{LG}(\sim 30 \%)$ and channel of $2.6 \mu \mathrm{m}$ length covered by $1 \mathrm{LG}(\sim 56 \%), 2 \mathrm{LG}$ $(\sim 30 \%)$ and 3LG (14\%) was obtained (see Figure 5 for SKPM map of the device in the main text). Residues due to contamination from resists and solvents as a result of the fabrication process have been removed by mechanically scraping the residues from side-to-side using soft cantilevers in contact-mode AFM to avoid damaging the graphene device $[32,33]$. Figure 5a in the manuscript shows the SKPM map of the device following the cleaning process.

6. Transport measurements: The transport properties of the device were investigated using the AC Hall effect and 4-point resistance measurements. The AC Hall effect was investigated by applying an out-of-plane AC magnetic field $\left(B_{A C}=5 \mathrm{mT}\right.$ at $\left.126 \mathrm{~Hz}\right)$ [34]. The resulting transverse $\mathrm{AC}$ signal of the $\mathrm{DC}$ current biased $\left(I_{\text {bias }}=50 \mu \mathrm{A}\right)$ Hall bar device contains contributions from the AC Hall voltage (in-phase) and inductance effects (90 out-of-phase). Using Stanford Research Systems SR830 lock-in amplifiers and the $I_{\text {bias }}$ reversal technique, the AC Hall voltage $\left(V_{H}\right)$ was accurately separated by measuring only the in-phase component of the AC signal at $+/-I_{\text {bias }}$. The resistance of the graphene channel $\left(R_{4}\right)$ was determined using the 4-point technique, i.e., by applying $+/-I_{\text {bias }}$ and measuring the voltage drop from cross 1 to cross 2 . The current reversal during the $R_{4}$ measurement eliminates the thermal electric voltage offset. From these measurements, the carrier density $\left(n=\frac{I_{b i a s} B_{A C}}{e V_{H}}\right)$ and carrier mobility 
$\left(\mu=\frac{L}{W} \frac{1}{e n R_{4}}\right)$ were obtained, where $e$ is the electronic charge and L/W is the device length/width ratio $(\sim 3.52)$.

\section{REFERENCES}

1. Schedin, F., Geim, A., Morozov, S. V, Hill, E. W., Blake, P., Katsnelson, M. I., \& Novoselov, K. S. (2007). Detection of individual gas molecules adsorbed on graphene. Nature Materials, 6(9), 652-5. doi:10.1038/nmat1967.

2. Dan, Y., Lu, Y., Kybert, N. J., Luo, Z., \& Johnson, A. T. C. (2009). Intrinsic Response of Graphene Vapor Sensors. Nano Letters 9, 1472-1475.

3. Fowler, J. D., Allen, M. J., Tung, V. C., Yang, Y., Kaner, R. B., Weiller, B. H. (2009). Practical chemical sensors from chemically derived graphene. ACS Nano 3, 301-306.

4. Castro Neto, A. H., Peres, N. M. R., Novoselov, K. S., \& Geim, A. K. (2009). The electronic properties of graphene. Reviews of Modern Physics, 81(1), 109-162. doi:10.1103/RevModPhys.81.109

5. Freitas, R. R. Q., Rivelino, R., Mota, F. D. B., \& Castilho, C. M. C. De. (2011). DFT Studies of the Interactions of a Graphene Layer with Small Water Aggregates. J. Phys. Chem. A, 12348-12356.

6. Berashevich, J., \& Chakraborty, T. (2009). Tunable band gap and magnetic ordering by adsorption of molecules on graphene. Physical Review B, 80(3), 033404. doi:10.1103/PhysRevB.80.033404

7. Voloshina, E., Usvyat, D., Schutz, M., Dedkov, Y., \& Paulus, B. (2011). On the physisorption of water on graphene: a $\operatorname{CCSD}(\mathrm{T})$ study. Phys. Chem. Chem. Phys. 13(25), 12041-12047. doi:10.1039/C1CP20609E 
8. Kalon, G., Shin, Y. J., \& Yang, H. (2011). Tunable metal-insulator transitions in bilayer graphene by thermal annealing. Applied Physics Letters, 98(23), 233108. doi:10.1063/1.3598403

9. Yang, Y., Brenner, K., \& Murali, R. (2012). The influence of atmosphere on electrical transport in graphene. Carbon, 50(5), 1727-1733. doi:10.1016/j.carbon.2011.12.008

10. Yavari, F., Kritzinger, C., Gaire, C., Song, L., Gulapalli, H., Borca-Tasciuc, T., Koratkar, N. (2010). Tunable bandgap in graphene by the controlled adsorption of water molecules. Small (Weinheim an Der Bergstrasse, Germany), 6(22), 2535-8. doi:10.1002/smll.201001384

11. Chen, J.-H., Jang, C., Adam, S., Fuhrer, M. S., Williams, E. D., \& Ishigami, M. (2008). Charged-impurity scattering in graphene. Nature Physics, 4(5), 377-381. doi:10.1038/nphys935

12. Lohmann, T., Klitzing, K. Von, \& Smet, J. H. (2009). Four-Terminal MagnetoTransport in Graphene p-n Junctions Created by Spatially Selective Doping. Nano Letters, 9(5), 1973. doi: 10.1021/n1900203n

13. Lafkioti, M., Krauss, B., Lohmann, T., Zschieschang, U., Klauk, H., Klitzing, K. V, \& Smet, J. H. (2010). Graphene on a hydrophobic substrate: doping reduction and hysteresis suppression under ambient conditions. Nano Letters, 10(4), 1149-53. doi:10.1021/n1903162a

14. Sidorov, A. N., Gaskill, K., Buongiorno Nardelli, M., Tedesco, J. L., Myers-Ward, R. L. et al. (2012). Charge transfer equilibria in ambient-exposed epitaxial graphene on (0001) 6 H-SiC. Journal of Applied Physics, 111(11), 113706. doi:10.1063/1.4725413

15. Huard, B., Stander, N., Sulpizio, J., \& Goldhaber-Gordon, D. (2008). Evidence of the role of contacts on the observed electron-hole asymmetry in graphene. Physical Review B, 78(12), 121402. doi:10.1103/PhysRevB.78.121402 
16. Ribeiro, R., Peres, N., Coutinho, J., \& Briddon, P. (2008). Inducing energy gaps in monolayer and bilayer graphene: Local density approximation calculations. Physical Review B, 78(7), 075442. doi:10.1103/PhysRevB.78.075442

17. Leenaerts, O., Partoens, B., \& Peeters, F. (2009). Water on graphene: Hydrophobicity and dipole moment using density functional theory. Physical Review B, 79(23), 1-5. doi:10.1103/PhysRevB.79.235440

18. V. Panchal, et al. unpublished results

19. Wehling, T. O., Lichtenstein, A. I., \& Katsnelson, M. I. (2008). First-principles studies of water adsorption on graphene: The role of the substrate. Applied Physics Letters, 93(20), 202110. doi:10.1063/1.3033202

20. Datta, S. S., Strachan, D. R., Mele, E. J., \& Johnson, A. T. C. (2009). Surface Potentials and Layer Charge Distributions in Few-Layer Graphene Films. Nano Letters, 9(1), 7.

21. Kazakova, O., Burnett, T. L., Patten, J., Yang, L., \& Yakimova, R. (2013). Epitaxial graphene on $\mathrm{SiC}(0001)$ : functional electrical microscopy studies and effect of atmosphere. Nanotechnology, 24(21), 215702. doi:10.1088/0957-4484/24/21/215702

22. Panchal, V., Pearce, R., Yakimova, R., Tzalenchuk, A., \& Kazakova, O. (2013). Standardization of surface potential measurements of graphene domains. Scientific Reports, 3(2), 2597. doi:10.1038/srep02597

23. Ferrari, A. C., Meyer, J. C., Scardaci, V., Casiraghi, C., Lazzeri, M., Mauri, F., Geim, A. K. (2006). Raman Spectrum of Graphene and Graphene Layers. Physical Review Letters, 97(18), 1-4. doi:10.1103/PhysRevLett.97.187401

24. Ryu, S., Liu, L., Berciaud, S., Yu, Y.-J., Liu, H., Kim, P., ... Brus, L. E. (2010). Atmospheric Oxygen Binding and Hole Doping in Deformed Graphene on a $\mathrm{SiO}(2)$ Substrate. Nano Letters, 4944-4951. doi:10.1021/nl1029607

25. Yu, Y., Zhao, Y., Ryu, S., Brus, L. E., Kim, K. S., \& Kim, P. (2009). Tuning the Graphene Work Function by Electric Field Effect, Nano Letters, 9 (10), 3430-3434 
26. Kim, J.-H., Hwang, J. H., Suh, J., Tongay, S., Kwon, S., Hwang, C. C., ... Young Park, J. (2013). Work function engineering of single layer graphene by irradiation-induced defects. Applied Physics Letters, 103(17), 171604. doi:10.1063/1.4826642

27. Levesque, P. L., Sabri, S. S., Aguirre, C. M., Guillemette, J., Siaj, M., Desjardins, P., ... Martel, R. (2011). Probing charge transfer at surfaces using graphene transistors. Nano Letters, 11(1), 132-7. doi:10.1021/nl103015w

28. Tadich, A., Edmonds, M. T., Ley, L., Fromm, F., Smets, Y., Mazej, Z., ... Wanke, M. (2013). Tuning the charge carriers in epitaxial graphene on $\mathrm{SiC}(0001)$ from electron to hole via molecular doping with C60F48. Applied Physics Letters, 102(24), 241601. doi:10.1063/1.4811248

29. Liscio, A., Palermo, V., Mu, K., \& Samori, P. (2008). Tip - Sample Interactions in Kelvin Probe Force Microscopy: Quantitative Measurement of the Local Surface Potential, The Journal of Physical Chemistry C 112 (44), 17368-17377.

30. Schenk, M., Füting, M., \& Reichelt, R. (1998). Direct visualization of the dynamic behavior of a water meniscus by scanning electron microscopy. Journal of Applied Physics, 84(9), 4880. doi:10.1063/1.368731

31. Li, Z., Wang, Y., Kozbial, A., Shenoy, G., Zhou, F., McGinley, R., ... Liu, H. (2013). Effect of airborne contaminants on the wettability of supported graphene and graphite. Nature Materials, 12(10), 925-31. doi:10.1038/nmat3709

32. Panchal, V., Lartsev, A., Manzin, A., Yakimova, R., Tzalenchuk, A., \& Kazakova, O. (2014). Visualisation of edge effects in side-gated graphene nanodevices. Scientific Reports, 4, 5881. doi:10.1038/srep05881

33. Kazakova, O., Panchal, V., \& Burnett, T. (2013). Epitaxial Graphene and GrapheneBased Devices Studied by Electrical Scanning Probe Microscopy. Crystals, 3(1), 191233. doi:10.3390/ 
34. Tousson, E., \& Ovadyahu, Z. (1998) Anomalous field dependence of the Hall coefficient in disordered metals. Physical Review B, 38(17), 12290-12297. doi:10.1103/PhysRevB.38.12290

35. Latil, S., Henrard, L. (2006). Charge Carriers in Few-Layer Graphene Films. Physical Review Letters, 97(3), 036803. doi:10.1103/PhysRevLett.97.036803

36. Ohta, T., Bostwick, A., McChesney, J., Seyller, T., Horn, K., \& Rotenberg, E. (2007). Interlayer Interaction and Electronic Screening in Multilayer Graphene Investigated with Angle-Resolved Photoemission Spectroscopy. Physical Review Letters, 98(20), 206802. doi:10.1103/PhysRevLett.98.206802

37. Koshino, M. (2010). Interlayer screening effect in graphene multilayers with ABA and ABC stacking. Physical Review B, 81(12), 125304. doi:10.1103/PhysRevB.81.125304

38. Nyakiti, L.O., Wheeler, V.D., Garces, N.Y., Myers-Ward, R.L., Eddy C.R., Jr., Gaskill, D.K. (2012). Enabling graphene-based technologies: Toward wafer-scale production of epitaxial graphene. MRS Bulletin, 37(12), 1149-57.

39. Ni, Z.H., Wang, H.M., Luo, Z.Q., Wang, Y.Y., Yu,T., Wu, Y.H., Shen, Z.X., J. Raman Spectrosc. (2010). The effect of vacuum annealing on graphene 41, 479-483. DOI $10.1002 / j r s .2485$

40. Yang,Y., Huang,L.-I., Fukuyama, Y., Liu, F.-H., Real, M.A., Barbara,P., Liang, C.-T., Newell, D.B., Elmquist, R.E., Small (2015). Low carrier density epitaxial graphene devices on SiC, 11, No. 1, 90-95. DOI: 10.1002/smll.201400989

41. V. Panchal, K. Cedergren, R. Yakimova, A. Tzalenchuk, S. Kubatkin, O. Kazakova, J. Appl. Phys. 2012, 111, 07E509.

ASSOCIATED CONTENT: Surface potential values for 1, 2 and 3LG with the change in environment and adhesion measurements of the test sample are included in the Supplementary Information section. 


\section{Supplementary Information}

Surface potential measurements for one-, two- and three-layer graphene $(1,2$, and $3 \mathrm{LG})$ show the same trend described by the carrier concentration with the change in environment (Figure $5 \mathrm{~b}$ in the manuscript). The surface potential values given in Table $\mathrm{S} 1$ are plotted in Figure S1:

Table S1: Surface potential values of 1, 2, and 3LG for each environment extracted from images presented in Figure 2 in the manuscript.

\begin{tabular}{|c|c|c|c|}
\hline Environment & $\begin{array}{l}\text { SP }_{1 L G} / \mathrm{eV} \\
( \pm 0.020 \mathrm{eV})\end{array}$ & $\begin{array}{l}\mathrm{SP}_{2 L G} / \mathrm{eV} \\
( \pm 0.020 \mathrm{eV})\end{array}$ & $\begin{array}{l}\text { SP }_{3 L G} / \mathrm{eV} \\
( \pm 0.020 \mathrm{eV})\end{array}$ \\
\hline Ambient & -0.691 & -0.582 & -0.582 \\
\hline Vacuum & -0.429 & -0.48 & -0.492 \\
\hline $\begin{array}{l}\text { Vacuum post- } \\
\text { annealing }\end{array}$ & -0.21 & -0.271 & -0.301 \\
\hline $\mathbf{N}_{2}$ & -0.41 & -0.468 & -0.488 \\
\hline $10 \% \mathrm{RH}$ & -0.453 & -0.5 & -0.55 \\
\hline $20 \%$ RH & -0.454 & -0.5 & -0.548 \\
\hline $30 \% \mathrm{RH}$ & -0.46 & -0.495 & -0.544 \\
\hline $40 \%$ RH & -0.469 & -0.494 & -0.542 \\
\hline $50 \%$ RH & -0.476 & -0.49 & -0.537 \\
\hline $60 \%$ RH & -0.489 & -0.476 & -0.522 \\
\hline $70 \%$ RH & -0.492 & -0.459 & -0.504 \\
\hline
\end{tabular}




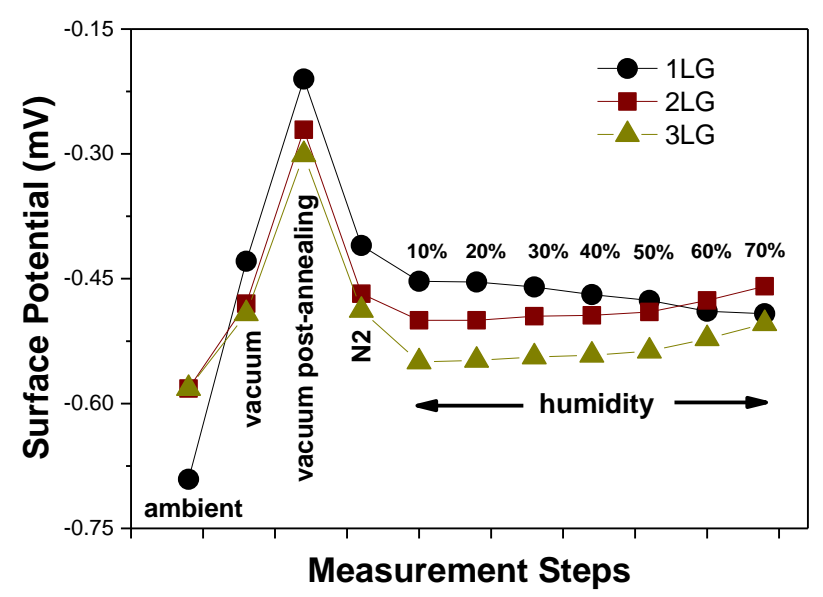

Figure S1: Surface potential for 1, 2 and 3LG with the change in environment, from ambient to vacuum, and to increasing humidity levels.

Table S2: Work function values derived from SKPM measurements on 1, 2 and 3LG exposed to ambient, vacuum, low and high humidity levels:

\begin{tabular}{cccc}
\hline Environment & $\boldsymbol{\Phi}_{1 L G}$ & $\Phi_{2 L G}$ & $\Phi_{3 L G}$ \\
& $(e V)$ & $(e V)$ & $(e V)$ \\
\hline Ambient & $4.79 \pm 0.02$ & $4.68 \pm 0.02$ & $4.69 \pm 0.02$ \\
Vacuum post- & $4.31 \pm 0.02$ & $4.37 \pm 0.02$ & $4.40 \pm 0.02$ \\
annealing & & & \\
$\mathbf{1 0 \%}$ RH & $4.55 \pm 0.02$ & $4.60 \pm 0.02$ & $4.65 \pm 0.02$ \\
$\mathbf{7 0 \%}$ RH & $4.59 \pm 0.03$ & $4.56 \pm 0.03$ & $4.6 \pm 0.03$ \\
\hline
\end{tabular}

Adhesion measurements of test samples: 
Adhesion force mapping on test samples consisting of a pattern of hydrophobic octadecyltrichlorosilane (OTS) areas and hydrophilic gold areas, shown in Figure S2(a), confirmed the larger adhesion force on hydrophobic areas (Figure S2b). The histogram associated with the adhesion map shows the range of pull-off forces associated with OTS and $\mathrm{Au}$ areas (Figure S2c).
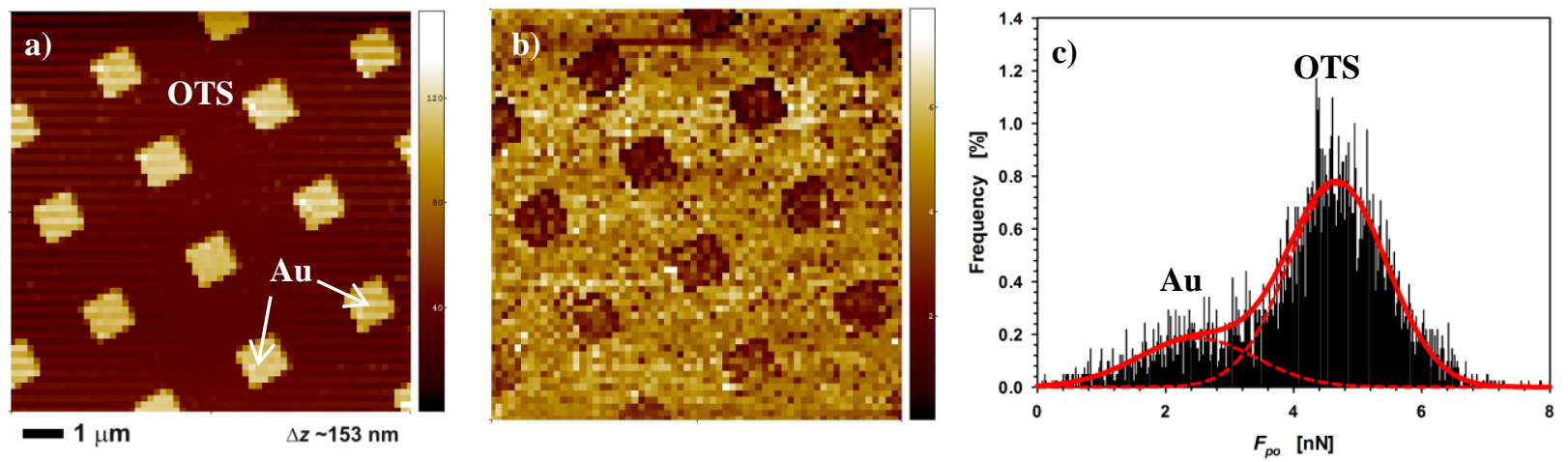

Figure S2: (a) Height map of OTS and Au pattern, (b) Corresponding adhesion map, (c) Histogram of the adhesion map with the Gaussian fits to the peaks indicated by red lines.

ACKNOWLEDGEMENT: The work was supported by the NMS under IRD Graphene Project, Graphene Flagship (No. CNECT-ICT-604391). Work at the Naval Research Laboratory was supported by the Office of Naval Research. 